\title{
Solar cycles in global temperatures
}

\author{
T. V. Kuznetsova and L. B. Tsirulnik \\ IZMIRAN, Russian Academy of Sciences, Moscow region, Troitsk, 142190, Russia \\ email: tvkuz@izmiran.ru
}

\begin{abstract}
We study variations of solar activity (sunspot numbers W) and anomaly of global temperature (GT) to detect a connection of their time variations on different time scales (including trends) to understand cause of global warming. We use a method of non-linear spectral analysis that is capable of making a self-consistent selection of trends, non-stationary oscillations and identifiing time intervals of development of non-linear processes (appearence of oscillations of large amplitude) in data. Analysis shows that trends of both spectra contribute main part to the data changes and show correlated increasing of W and GT during the studied interval. Cause of Maunder Minimum and present global warming is regime change (from epoch of damping to epoch of building up) of the powerfull oscillations at $\mathrm{T}=1000 \mathrm{yr}$. from the GT spectrum. Besides, time changes of GT and W has different type of connection in different range of periods $\mathrm{T}$ : non-stationary oscillations from the spectra of $\mathrm{W}$ and $\mathrm{GT}$ at $\mathrm{T}=10 \mathrm{yr}$. and $\mathrm{T}=30 \mathrm{yr}$. vary in opposite phase, at $\mathrm{T}=22 \mathrm{yr}$. vary in phase. The latter means different influence of odd and even 11-yr solar cycles on anomaly of GT (warming and cooling accordingly).
\end{abstract}

Keywords. Sun: solar-terrestrial relations

Owing to the transient character of some signals within solar and climate data, they cannot be well-characterized by any traditional method of spectral analysis. We use a method of a non-linear spectral analysis named by us a method of global minimum (MGM). MGM is capable of making a self-consistent selection of trends (polynomials or sinusoids) from a data set and singling out non-stationary sinusoids (with varying phase and amplitude). MGM and technique of removing a white noise from original time series is described by Tsirulnik et al. 1997. We use data of annual sunspot numbers $\mathrm{W}$ for 1700 - 2003; annual anomalies of Global Temperature (GT) for the period 1000 - 1990 obtained from proxy data (Jones et al. 1998). Spectral peaks of the calculated spectra have confidence statistical level higher than $95 \%$.

Figure 1 shows time variations of the most power and long-periodic non-stationary oscillations at period $\mathrm{T}=1000 \mathrm{yr}$. from the spectrum of anomaly of GT and variations of its amplitude for analysed years (extrapolation from 1990). The trend from the MGM spectrum of $\mathrm{W}$ is not shown at a figure. Trends (the most long-periodic parts of the spectra of W and GT) show synchronous increasing for 1700-2000. The trends have the highest power in both spectra that point to the main contribution of long-term variations to the data changes. Figure 1 shows how cycle at $\mathrm{T}=1000 \mathrm{yr}$ displays epoch of damping (i.e. of amplitude decrease) and of building up (of amplitude increase) of the oscillations. Such features are typical of non-linear oscillations. Values of anomaly of GT at figure 1 (a) are higher than ones at Medieval Warming Period $(t=1000 \mathrm{yr}$.) beginning from 1900 (appearance of positive anomaly); Maunder Minimum at $\mathrm{t}=1670 \mathrm{yr}$. is the first minimum after the regime change at $\mathrm{t} \sim 1250 \mathrm{yr}$, time of the minimum at figure 1 (b). The time interval at $\mathrm{t} \sim 1250 \mathrm{yr}$. is special time of the Earth's orbital motion: the planet passed its perihelion during winter solstice (previous such coincidence was in $20000 \mathrm{yr}$. $\mathrm{BC}$ ) that points to astronomical forcing of the regime change of the oscillations at $\mathrm{T}=$ $1000 \mathrm{yr}$, physical basis is presented by Kuznetsova et al. 2004 . 

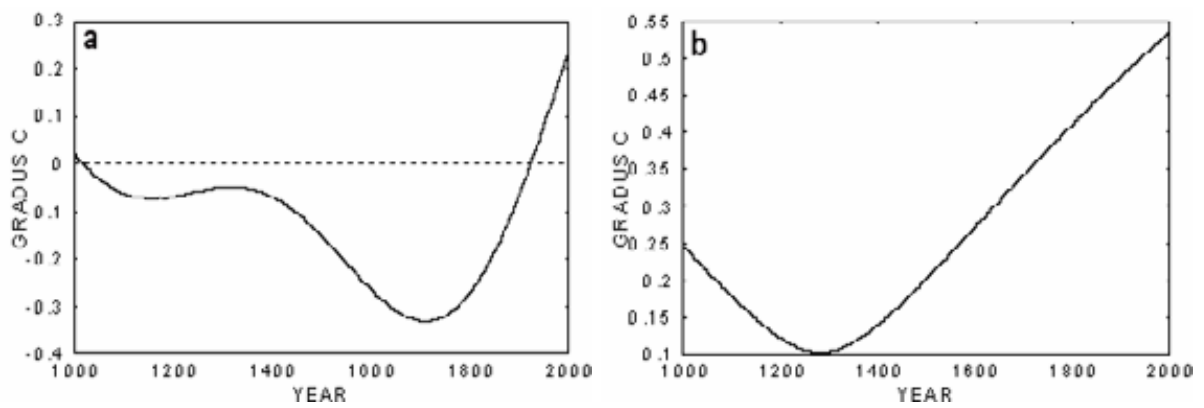

Figure 1. Time variations of non-stationary sinusoid at period $\mathrm{T}=1000 \mathrm{yr}$. from the MGM spectrum of anomaly of global temperature (a) and time changes of its amplitude (b).
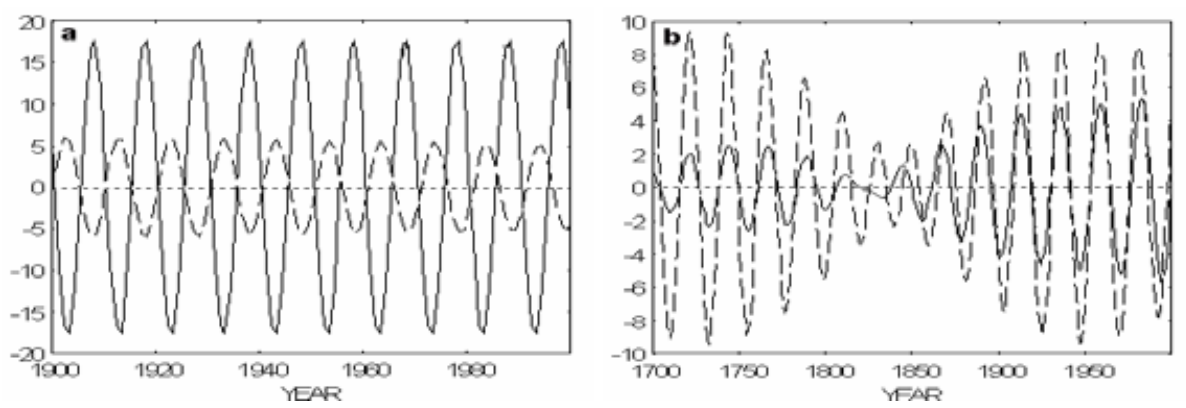

Figure 2. Time changes of non-stationary oscillations from the MGM spectra of anomaly of global temperature and sunspot numbers $\mathrm{W}$ at periods $\mathrm{T}=10 \mathrm{yr}$. (a) and $\mathrm{T}=22 \mathrm{yr}$. (b).

Connection of variations of $\mathrm{W}$ and GT has different character on different time scales that points to different physical mechanisms. In particular, time changes of powerful cycles at periods $\mathrm{T}=30 \mathrm{yr}$ and $\mathrm{T}=10 \mathrm{yr}$ in $\mathrm{W}$ and $\mathrm{GT}$ vary in opposite phase. From the other hand, the 22-yr non-stationary oscillations from both spectra (W and GT) vary in phase including time intervals of regime changes. Figure 2 presents time changes of the 22-year and 10-year cycles from the spectra of anomaly of GT and W; for comparison of their variations we use relative units. Analysis shows that even 11-yr solar cycles correspond to cooling GT, but odd cycles correspond to warming. This result about disparity of the 11-yr cycles is not taken usually into account by researches. The fact that 22-yr cycle reflects asymmetry of solar activity relative to sign of the solar magnetic field points to a physical mechanism: interaction of solar and terrestrial magnetic fields. Besides, oscillations of the 22-yr cycle in anomalies of angular velocity of the Earth and GT vary in phase that allowed us to explain the 22-yr variations of GT by atmosphere influence. However, cycle at $\mathrm{T}=22 \mathrm{yr}$. has relative small power in both spectra. Powerful cycles in the spectra ( this mechanism: an additional physical mechanism should be elaborated.

The work is supported by Russian Fund of Basic Researches, grant 06-05-64998.

\section{References}

Jones, P.D., Briffa, K.R., Barnett, T.P., \& Tett, S.F.B. 1998, World Data Center-A for Paleoclimatology, Series \# 1998-039. NOAA/NGDC

Kuznetsova, T. V. \& Tsirulnik, L.B. 2004, Adv. in Space Res., 34, 426

Tsirulnik, L.B., Kuznetsova, T.V., \& Oraevsky, V.N. 1997, Planet. and Space Sci., 44, 241 